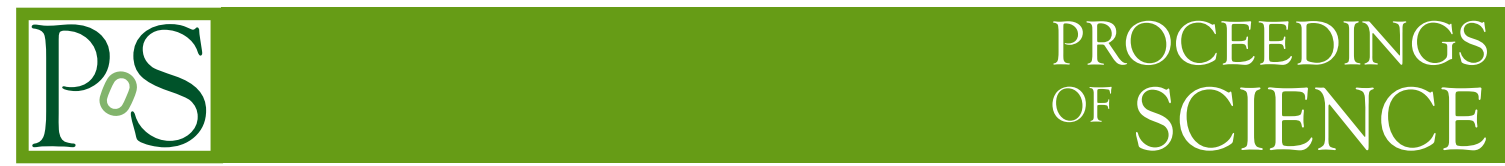

\title{
Search for Double Partons at CDF
}

\author{
Lee Pondrom* for the CDF Collaboration \\ University of Wisconsin, Madison \\ E-mail: pondromefnal.gov
}

A search for double parton hard scattering has been performed using the $\mathrm{Z} \rightarrow \mu^{+} \mu^{-}$sample of the CDF detector corresponding to $9 \mathrm{fb}^{-1}$ of $\overline{\mathrm{p}}$ collision data taken in Run 2 at $1.96 \mathrm{TeV}$. The transverse region in $\phi$ relative to $\mathrm{p}_{\mathrm{TZ}}$, defining the 'underlying event', is used to look for a jet pair from a second hard scatter. No back-to-back dijet excess is seen in 45,633 Z events with $\mathrm{p}_{\mathrm{TZ}}>10$ $\mathrm{GeV} / \mathrm{c}$ and at least one jet with $\mathrm{E}_{\mathrm{T}}>5 \mathrm{GeV}$.

XXI International Baldin Seminar on High Energy Physics Problems September 10-15, 2012

JINR, Dubna, Russia

${ }^{*}$ Speaker. 

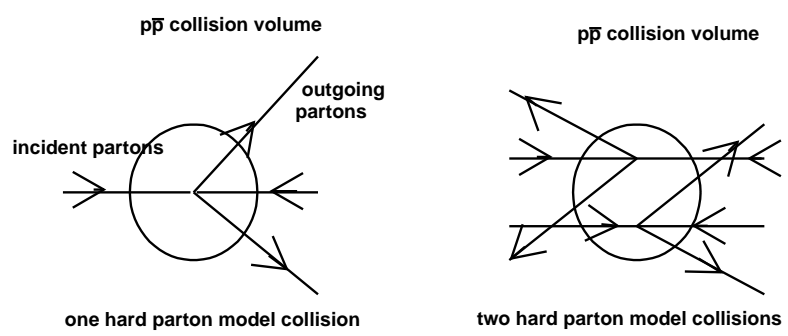

Figure 1: Schematic diagrams of single parton (left) and double parton (right)scattering in the same $\bar{p} p$ collision.

\section{Introduction}

The CDF collaboration has published a study of the 'underlying event' in the $\mathrm{Z} \rightarrow 1^{+} 1^{-}$data produced by $\bar{p}$ p collisions at $1.96 \mathrm{TeV}$. [1] A result of this study is a tune of the Monte Carlo simulation program PYTHIA [2] to reproduce the observed underlying event activity. The number of secondary or rescattering non-perturbative interactions is one of the parameters adjusted in the tune.

Double parton interactions are a natural extension of the parton model. The parton model is an essential ingredient of Monte Carlo simulation programs like PYTHIA, and has enjoyed much success in describing high energy hadron collisions as parton-parton $2 \rightarrow 2$ scattering folded into parton distributions determined by lepton-hadron scattering. In a double parton interaction this process occurs twice in the same $\bar{p}$ p collision. Figure 1 shows schematically the single and double parton processes. The two hard scatters are perturbative, and are independent, consistent with the conservation laws. If the momentum fractions and energies involved in the two scatters are modest, independence should obtain. Therefore, a second $2 \rightarrow 2$ scatter resulting in two back to back jets in the final state independent of the primary interation is a signature of double parton interactions (DPI). Such a process can be modeled by two separate interactions (vertices) in the same event. A recent study by the D0 Collaboration reviews previous work in this subject. [3]

\section{Apparatus}

The CDF Run 2 detector has been described elsewhere [4]. Vital components of the CDF detector for this analysis were the central charged particle tracker, called the COT, the central electromagnetic and hadronic calorimetry, called CEM and CHA respectively, and the central muon detectors. The origin of the right-handed CDF coordinate system was at the center of the detector, on the beam line, with $\hat{z}$ in the proton direction, $\hat{x}$ in the horizontal plane, and $\hat{y}$ directed vertically upwards. The azimuthal angle $\phi$ was measured in the xy plane, and the polar angle $\theta$ was used to define the pseudorapidity $\eta=-\log (\tan (\theta / 2))$. Events were restricted to the central region $(|\eta|<1)$, where the tracking efficiency was high. The COT was a drift chamber in a $1.4 \mathrm{~T}$ magnetic field parallel to $\hat{z}$, with an inner radius of $40 \mathrm{~cm}$ and an outer radius of $140 \mathrm{~cm}$, containing 30,000 sense wires. The single charged track momentum resolution, including the vertex constraint in the 


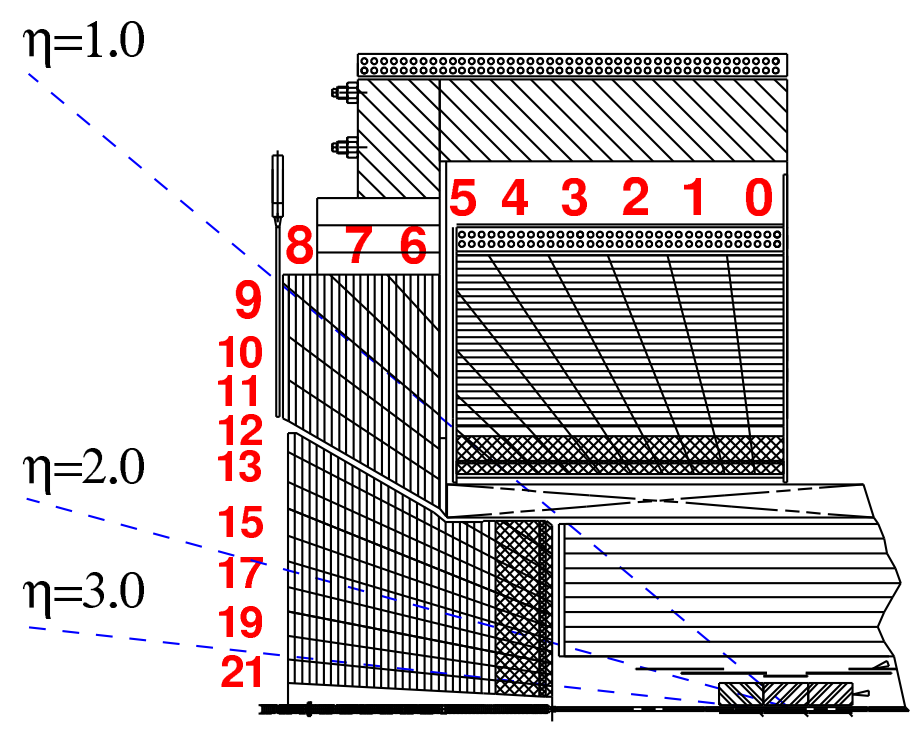

Figure 2: CDF calorimetry tower configuration. Towers 0-5 are in the CHA; towers 6 and 7 are shared between the CHA and the WHA (endwall); towers 8-11 are WHA, and 21-21 are PHA (Plug).The CMU muon chambers are just outside the CHA, inside the return iron, while the CMP chambers are outside the return iron.

xy plane, was $\delta \mathrm{p}_{\mathrm{T}} / \mathrm{p}_{\mathrm{T}}^{2}=0.001(\mathrm{GeV} / \mathrm{c})^{-1}$, or $\delta \mathrm{p}_{\mathrm{T}} / \mathrm{p}_{\mathrm{T}}=0.05$ at $50 \mathrm{GeV} / \mathrm{c}$. The z vertex resolution for an extrapolated COT track was $\delta \mathrm{z}=3 \mathrm{~mm}$.

The CEM and the CHA were sampling calorimeters, with lead plates and iron plates respectively, interspersed with scintillator. The CEM had 31 lead plates each $0.32 \mathrm{~cm}$ thick, and 0.5 $\mathrm{cm}$ scintillator, for a total of 18 radiation lengths, while the CHA had 32 iron plates each 2.5 $\mathrm{cm}$ thick, and $1.0 \mathrm{~cm}$ scintillator. The total number of interaction lengths for pions penetrating the CEM+CHA was 5.5, a number relevant both for hadronic energy resolution and muon detection. The calorimeters were arranged in towers in $(\eta, \phi)$ space, with granularity $\delta \eta=0.1$ and $\delta \phi=0.26\left(15^{0}\right)$. A side view of $1 / 4$ of the tracking and calorimetry is shown in figure 2 . The energy resolution at $50 \mathrm{GeV}(\sigma / \mathrm{E})$ for CEM was $2 \%$, and for CHA was $11 \%$. The measured transverse energies were corrected using the standard jet energy corrections package. [5].

The central muon detectors had two components, called the CMU and the CMP. CMU was composed of a barrel of $4.52 \mathrm{~m}$ long drift chambers outside the central calorimeters, $3.5 \mathrm{~m}$ radial distance from the beam line, covering \pm 0.6 in $\eta$.To increase the absorber and improve the pion rejection a second set of drift chambers was installed outside the $60 \mathrm{~cm}$ thick iron return yoke on the top and bottom of the detector. Drift chambers were also installed on the sides of the detector, outside extra $60 \mathrm{~cm}$ thick iron plates. Muon candidates with tracks in the CMP traversed about 7.8 pion interaction lengths. The CMP was a square box outside the detector, with $\eta$ coverage matching the CMU. A good quality central muon candidate had momentum measured in the COT, and matched drift chamber stubs in both the CMU and CMP. Such muon candidates had negligible background. 


\section{Transverse tracks}

The transverse region in the $\phi$ plane perpendicular to the beams is defined by $\pi / 3<\Delta \phi<$ $2 \pi / 3$ relative to the highest $\mathrm{E}_{\mathrm{T}}$ jet in the jet data sample, or $\mathrm{p}_{\mathrm{TZ}}$ in the Drell-Yan sample with $\mathrm{Z} \rightarrow \mu^{+} \mu^{-}$, as shown in figure 3 . Figure 4 shows the scalar sum $\mathrm{p}_{\mathrm{T}}$ of all tracks in the transverse region for jet data, compared with the PYTHIA Monte Carlo event generator. The average value of the scalar sum $\mathrm{p}_{\mathrm{T}}$ of all charged tracks in the transverse region increases slowly with the leading $\mathrm{E}_{\mathrm{T}}$ in the event, and is the same for jets and Z's, where leading jet $\mathrm{E}_{\mathrm{T}} \simeq \mathrm{p}_{\mathrm{TZ}}$. The integrals over all $\mathrm{p}_{\mathrm{T}}$ of the curves in figure 4 , when properly normalized, agree with the data points shown in figure 4.1 of Ref[1].

By definition the transverse region is relatively quiet, with most of the energy lying parallel to the thrust axis of the leading jet. The transverse region should be a good place to look for DPI, if one can devise a method to select events with jet activity there. This suggests a new technique to look for DPI: Use high $\mathrm{p}_{\mathrm{T}}$ transverse tracks as a 'trigger' signature of a second hard interaction, by imposing the arbitrary requirement $\Sigma$ transtrackp $_{\mathrm{T}}>15 \mathrm{GeV} / \mathrm{c}$ as the trigger, and studying the jet activity in the transverse region of the 'triggered' events. Events with two separate vertices can be used to test the method.

\section{Jet events with two vertices}

The presence of more than one vertex per beam crossing depends on the luminosity, and on the definition of the second vertex. A total inelastic $\bar{p} p$ cross section of $60 \mathrm{mb}$ at $1.96 \mathrm{TeV}$, [6] a luminosity of $1 \mathrm{E} 32 \mathrm{~cm}^{-2} \mathrm{sec}^{-1}$, and a crossing frequency of $2.52 \mathrm{Mhz}$ [7] gives and average $\overline{\mathrm{n}}=2.4$ interactions per beam crossing. Most of these secondary vertices are not detected with the criteria defined below, because they do not produce high $\mathrm{p}_{\mathrm{T}}$ central tracks. The interaction region in the $\mathrm{z}$ direction at CDF was Gaussian, with $\sigma=30 \mathrm{~cm}$, so that $2 / 3$ of the interaction vertices were within $\pm 30 \mathrm{~cm}$. The vertex resolution of the COT $(\sigma=3 \mathrm{~mm})$ resolved $99 \%$ of the extra vertices. The primary vertex was the jet trigger vertex, with jet $\mathrm{E}_{\mathrm{T}}>100 \mathrm{GeV}$. The secondary vertex was defined as having at least 3 charged tracks with $\mathrm{p}_{\mathrm{T}}>0.5 \mathrm{GeV} / \mathrm{c}$ and $|\eta|<1$, , separated from the primary vertex by at least $10 \mathrm{~cm}$. For the data sample used, $30 \%$ of the events had a secondary vertex satisfying these requirements. From this number and the run averaged instantaneous luminosity of $1 \mathrm{E} 32 \mathrm{~cm}^{-2} \mathrm{sec}^{-1}$ one obtains a cross section for the second vertex of $\sigma=12 \mathrm{mb}$. Figure 5 illustrates the procedure. The primary vertex, called vtx1 in the figure, has at least two jets, the highest $\mathrm{E}_{\mathrm{T}}$ jet being the trigger, and the second vertex is at least $10 \mathrm{~cm}$ away. The transverse region defined on vtx 1 is translated to vtx 2 , and the 'transverse tracks' are measured on vtx 2 . Tracks are used to define the vertices. A $100 \mathrm{GeV}$ jet contains several charged particles, so the definition of the vertex is unambiguous. Assigning a vertex to a low $\mathrm{E}_{\mathrm{T}}$ jet ( $10 \mathrm{GeV}$ for example) is problematic, because there may be no tracks associated with the jet.

Figure 6 shows the scalar sum $\mathrm{p}_{\mathrm{T}}$ spectrum of the transverse tracks on the second vertex, compared to the jet data shown in figure 4. Also shown is the fraction of transverse tracks with $\mathrm{p}_{\mathrm{T}}>15 \mathrm{GeV} / \mathrm{c}$ as a function of the $\mathrm{E}_{\mathrm{T}}$ of the leading jet or $\mathrm{Z}$. (The $\mathrm{Z}$ data are discussed below.) The fraction increases rapidly from $10^{-3}$ for 'minbias' to $10^{-2}$ for $\mathrm{p}_{\mathrm{TZ}}>10 \mathrm{GeV} / \mathrm{c}$. Note that jet data and $\mathrm{Z}$ data agree. The 'trigger' creates a pair of jets on the second vertex with $\mathrm{E}_{\mathrm{T}}>5 \mathrm{GeV}$ with a 


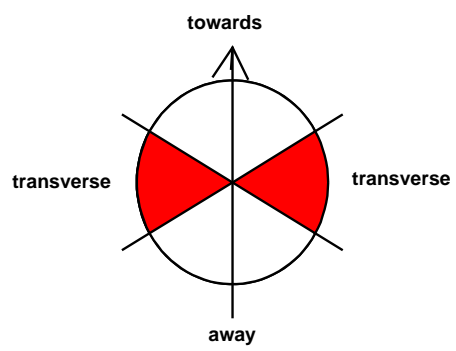

Figure 3: Diagram showing the transverse region in the xy plane. The area shown in red is $\pi / 3<\Delta \phi<2 \pi / 3$ relative to the leading jet or $\mathrm{Z}$.
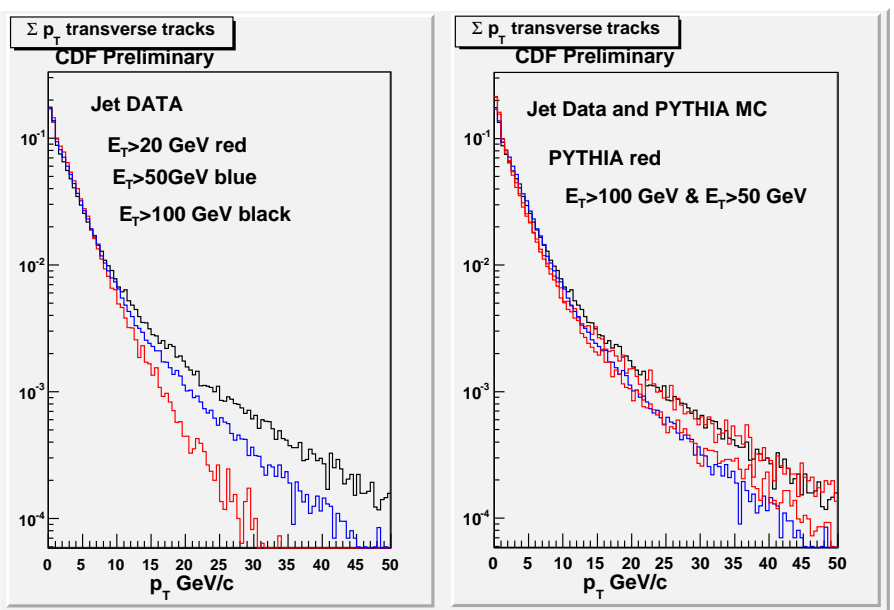

Figure 4: Scalar sum of $\mathrm{p}_{\mathrm{T}}$ of transverse charged tracks for dijet data on the left, and compared to PYTHIA on the right. The fraction at $\mathrm{p}_{\mathrm{T}}>15 \mathrm{GeV}$ increases with increasing jet $\mathrm{E}_{\mathrm{T}}$.

$60 \%$ efficiency, so the overall probability of finding a pair of jets on the second vertex is $6 \times 10^{-4}$, or a dijet cross section of $7 \mu \mathrm{b}$.

Figure 7 and figure 8 show the energies and azimuths of the jets created on the second vertex by the $\Sigma$ transtrackp $\mathrm{T}_{\mathrm{T}}>15 \mathrm{GeV} / \mathrm{c}$ 'trigger'. The extra jets appear in the transverse region in $\phi$, just like they are supposed to. Their average energies are softer than those from the jet20 trigger the lowest $\mathrm{E}_{\mathrm{T}} \mathrm{CDF}$ jet data sample. The key distribution is $\Delta \phi$ jets $3 / 4$, shown in figure 9 , which shows a clear back-to-back peak for the pair of independent jets created on the second vertex by the 'trigger'.

\section{5. $\mathrm{Z} \rightarrow \mu^{+} \mu^{-}$data sample}

The $\mathrm{Z} \rightarrow \mu \mu$ final state was chosen for the DPI search because of its high purity and relative simplicity. The entire CDF run 2 muon data sample, $\int L \mathrm{dt}=9 \mathrm{fb}^{-1}$, was scanned to select $\mathrm{Z} \rightarrow$ $\mu^{+} \mu^{-}$candidate events. The DPI search strategy was to use $\mathrm{p}_{\mathrm{TZ}}$ to define the transverse region in $\phi$. Only one vertex was allowed per event. The muon requirements were:

- Require two muons of opposite charge with $|\eta|<1$. and $\mathrm{p}_{\mathrm{T}}>20 \mathrm{GeV} / \mathrm{c}$. 


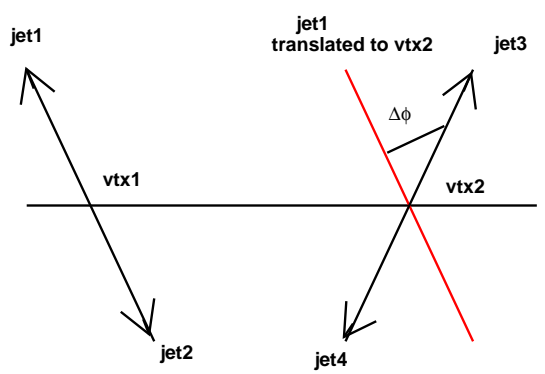

Figure 5: Diagram of the two vertex configuration. VTX1 is the primary vertex, where the $100 \mathrm{GeV}$ trigger jet resides, and VTX2 is the secondary vertex, defined by at least three charged tracks with $\mathrm{p}_{\mathrm{T}}>0.5 \mathrm{GeV} / \mathrm{c}$.

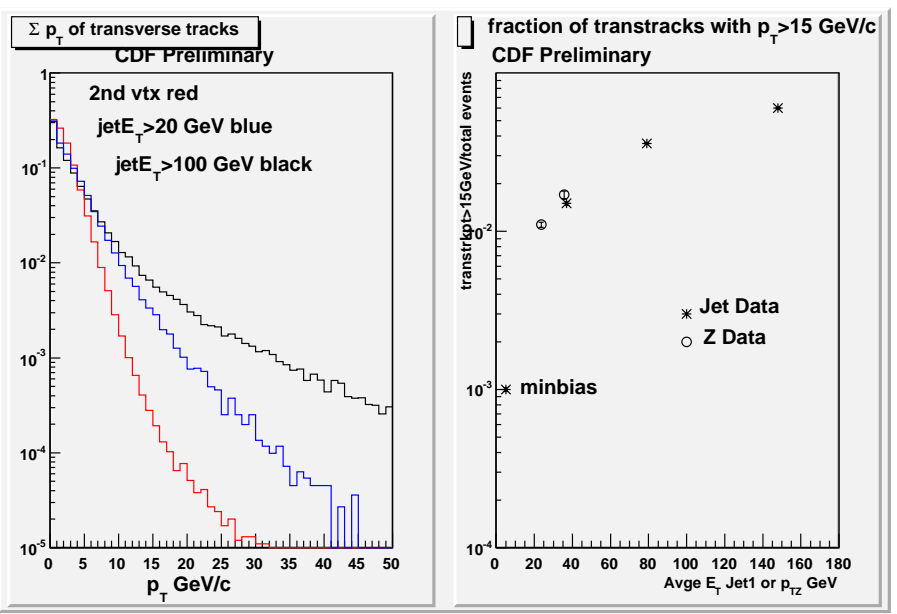

Figure 6: Left hand figure shows the scalar sum $\mathrm{p}_{\mathrm{T}}$ of transverse tracks for the secondary vertex, compared to the same plots for jet 20 and jet 100 . The secondary vertex is very soft, and is called 'minbias'. The right hand figure shows the fraction of events with scalar sum $\mathrm{p}_{\mathrm{T}}>15 \mathrm{GeV} / \mathrm{c}$ as a function of the highest jet $\mathrm{E}_{\mathrm{T}}$, or $\mathrm{p}_{\mathrm{TZ}}$. Note that the fractions for jets and Z's agree.

- Eliminate events with cosmic rays

- Require at least one good quality central muon satisfying CMU+CMP.

- 215589 events in $30<\mathrm{m}_{\mu \mu}<130 \mathrm{GeV}$ mass range

- 176351 events in $80<\mathrm{m}_{\mu \mu}<100 \mathrm{GeV}$ mass range - the Z's.

- 45633 events with at least one jet $\mathrm{E}_{\mathrm{T}}>5 \mathrm{GeV}$, and $\mathrm{p}_{\mathrm{TZ}}>10 \mathrm{GeV} / \mathrm{c}$.

The resulting mass and $\mathrm{p}_{\mathrm{T}}$ distributions for muon pairs are shown in figure 10, compared to the PythiA Monte Carlo including the CDF detector simulation. The agreement between the data and simulation is good. The simulation has a bit better mass resolution, and lies below the measured $\mathrm{p}_{\mathrm{TZ}}$ distribution above $50 \mathrm{GeV} / \mathrm{c}$.

Figures 11, 12, and 13 show the properties of the jet activity associated with $\mathrm{Z}$ events with $\mathrm{p}_{\mathrm{TZ}}>10 \mathrm{GeV} / \mathrm{c}$, compared to the PYTHIA simulation. 


\section{Transverse region in $\mathrm{Z}$ production}

Figure 14 shows the $\Sigma$ transtrackp $\mathrm{p}_{\mathrm{T}}$ distribution for $\mathrm{p}_{\mathrm{TZ}}>10 \mathrm{GeV} / \mathrm{c}$, data and PYTHIA, and jet1 $\mathrm{E}_{\mathrm{T}}$ distributions after the $\Sigma$ transtrackp $\mathrm{p}_{\mathrm{T}}>15 \mathrm{GeV} / \mathrm{c}$ 'trigger'. Figure 15 shows the effect of the 'trigger' on the azimuthal distributions of jets 1 and 2, both of which move into the transverse region. This is contrary to the expectations of DPI, where jet1, the highest $\mathrm{E}_{\mathrm{T}}$ jet, is supposed to balance the $\mathrm{p}_{\mathrm{TZ}}$. In fact, both jets 1 and 2 shift so as to form a triangle to balance the $\mathrm{Z}$, without any sign of DPI. This is shown in figure 16, where the 'trigger' has shifted the peak in $\Delta \phi$ jets 1-2 to about 2.3 radians, or $130^{\circ}$, which just fits the vectors into a $120^{\circ}$ triangle in the $\phi$ plane: $\left(\overrightarrow{\mathrm{p}}_{\mathrm{TZ}}, \overrightarrow{\mathrm{p}}_{\mathrm{Tj} 1}, \overrightarrow{\mathrm{p}}_{\mathrm{Tj} 2}\right)$. Figure 16 also shows that $\Delta \phi$ jets 2-3 has no inhancement near $\pi$ radians.

\section{DPI search}

If one assumes that there is one DPI of the type defined by the secondary vertex study, namely with three charged tracks $\mathrm{p}_{\mathrm{T}}>0.5 \mathrm{GeV} / \mathrm{c}$, and $|\eta|<1$., in each $\mathrm{Z}$ production event with $\mathrm{p}_{\mathrm{TZ}}>10$ $\mathrm{GeV} / \mathrm{c}$, then we expect $45633 \times 6 . \times 10^{-4}=27$ dijet events with a $\Delta \phi$ distribution like figure 9 . Figure 9 is replotted in figure 17 normalized to 27 events, together with the observed $\Delta \phi$ jets 2-3 (352 events) for comparison.

An alternate estimation method is based on figure 6 . The left hand plot shows that the scalar sum $\mathrm{p}_{\mathrm{T}}$ of transverse tracks is not independent of the energies involved in the main collision. The energy dependence of the fraction $\mathrm{p}_{\mathrm{T}}>15 \mathrm{GeV} / \mathrm{c}$ increases monotonically with jet $\mathrm{E}_{\mathrm{T}}$ or $\mathrm{p}_{\mathrm{TZ}}$. So the DPI contribution, if it exists, should be a decreasing fraction of the total as the $\mathrm{E}_{\mathrm{T}}$ of the event increases. If one assumes that the $\Sigma$ transversetrackp $\mathrm{p}_{\mathrm{T}}$ for Z's with $\mathrm{p}_{\mathrm{TZ}}>10 \mathrm{GeV} / \mathrm{c}$ contains within it a contribution from the DPI 'second vertex', then the fraction of the 352 dijets ( $2 \& 3)$ coming from DPI would be $0.001 / 0.012 \times 352=29$ events, in agreement with the above estimate.

In order to place a limit on the probability of the DPI vertex from the curves in figure 17 , it would be necessary to know with better accuracy the shape of $\Delta \phi 2 / 3$ from the primary vertex.

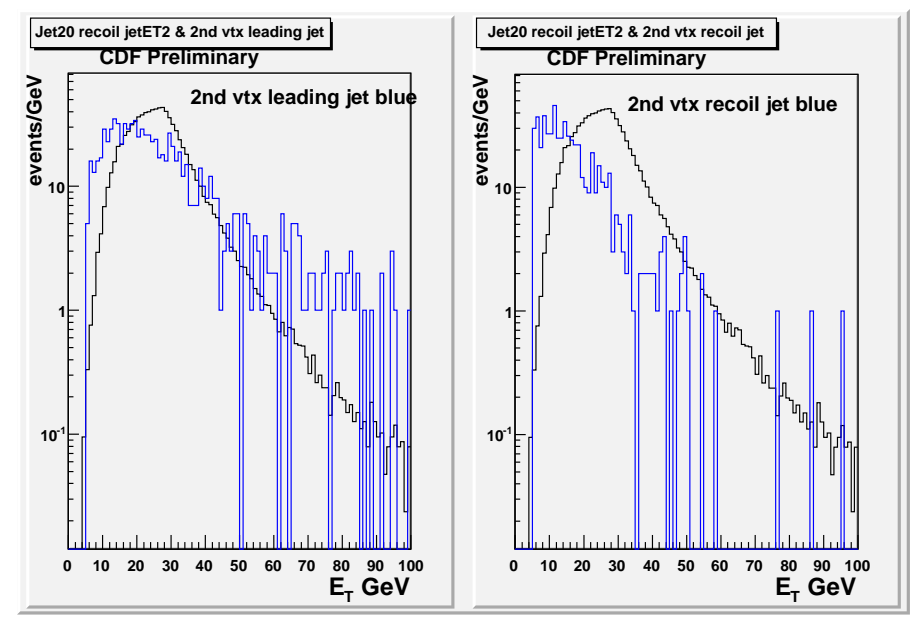

Figure 7: On the left is $\mathrm{E}_{\mathrm{T}}$ for jet3, and right for jet4, created by $\Sigma$ transtrack $\mathrm{p}_{\mathrm{T}}>15 \mathrm{GeV} / \mathrm{c}$ trigger on the second vertex. The energy distributions are compared to the recoil energy for a trigger jet $\mathrm{E}_{\mathrm{T}}>20 \mathrm{GeV}$. Jets 3 and 4 on average are softer than a jet20 trigger. 


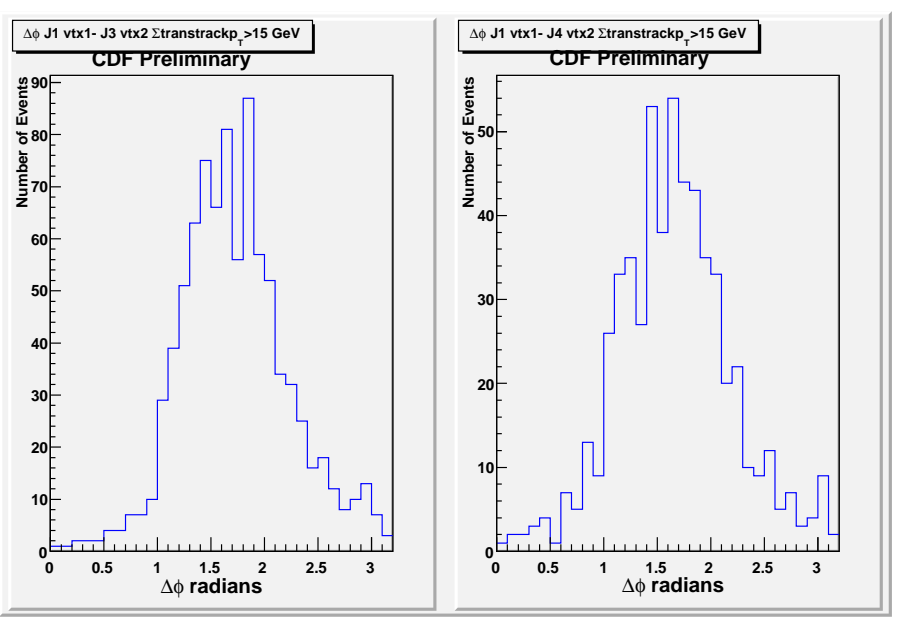

Figure 8: On the left is the azimuthal angle distribution of jet 3 on vertex 2 relative to jet1 on vertex 1 , and the same plot on the right for jet 4 / jet1. The 'trigger' does what it is supposed to do: it creates jets in the transverse region in $\phi$.

\section{Acknowledgements}

We thank the Fermilab staff and technical staffs of the participating institutions for their vital contributions. This work was supported by the U.S.Department of Energy and the National Science Foundation;the Italian Istituto Nazionale de fisica Nucleare; the Ministry of Education,Culture,Sports, Science and Technology of Japan;the Nautral Sciences and Engineering Research Council of Canada;the National Science Council of the Republic of China; the Swiss National Science Foundation; the A.P.Sloan Foundation;the Bundesministerium fur Bildund und Forschung, Germany; the Korean Science and Engineering Foundation and the Korean Research Foundation; the Science and Technology Facilities Council and the royal Society, UK; the Insti-

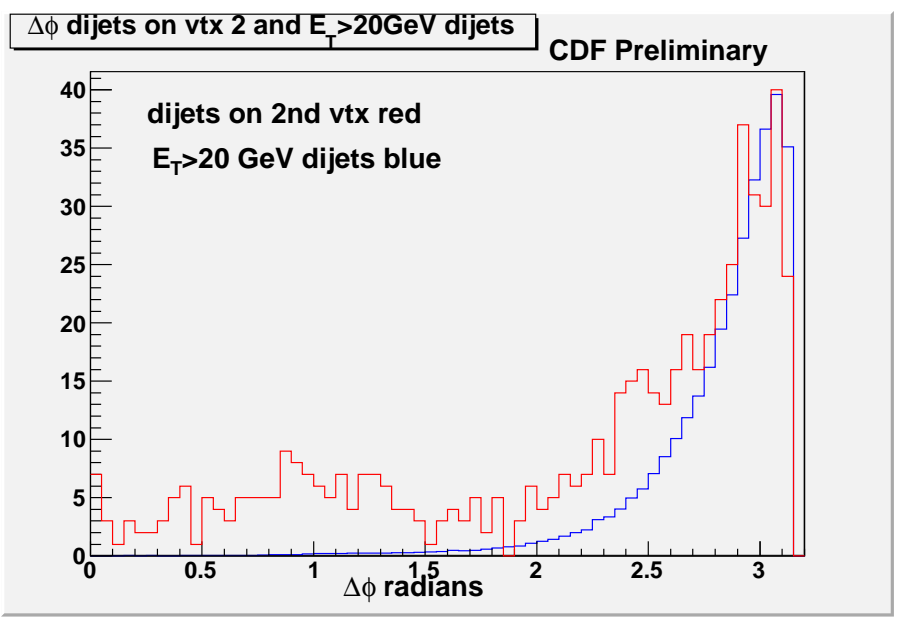

Figure 9: $\Delta \phi 34$ for the two jets on the second vertex created by the $\Sigma$ transtrackp $\mathrm{T}_{\mathrm{T}}>15 \mathrm{GeV} / \mathrm{c}$ 'trigger'. The back-to-back peak is compared to that for jet20 dijets, which have larger average $\mathrm{E}_{\mathrm{T}}$, and hence better angular resolution. 


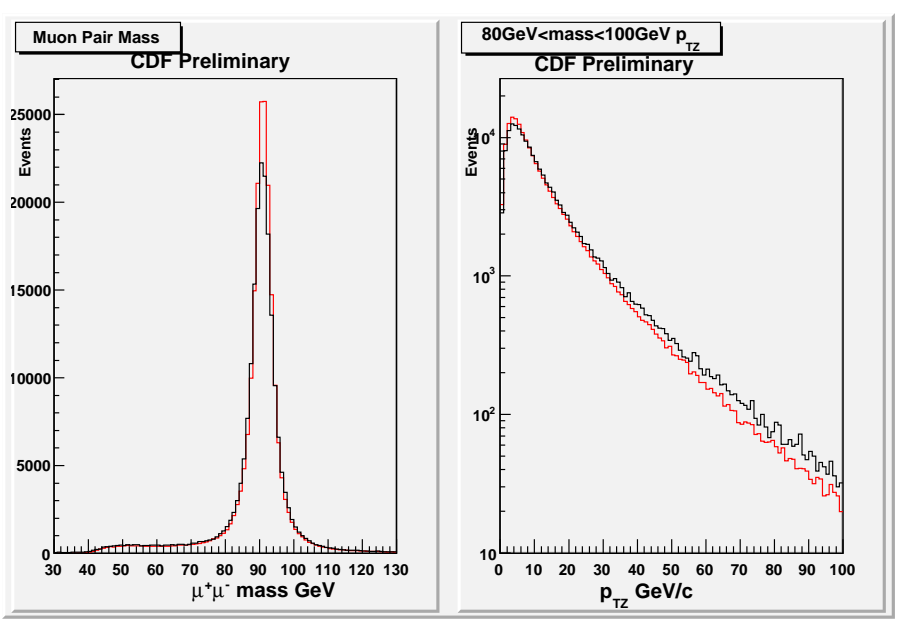

Figure 10: Left hand plot shows the $\mu^{+} \mu^{-}$pair mass data compared to PyTHIA Monte Carlo. The mass width for the data $\sigma=3.8 \mathrm{GeV}$ is slightly larger than the Monte Carlo. The natural width is $\Gamma_{Z}=2.5 \mathrm{GeV}$. The right hand plot compared the $\mathrm{p}_{\mathrm{TZ}}$ distributions in the $\mathrm{Z}$ mass range.

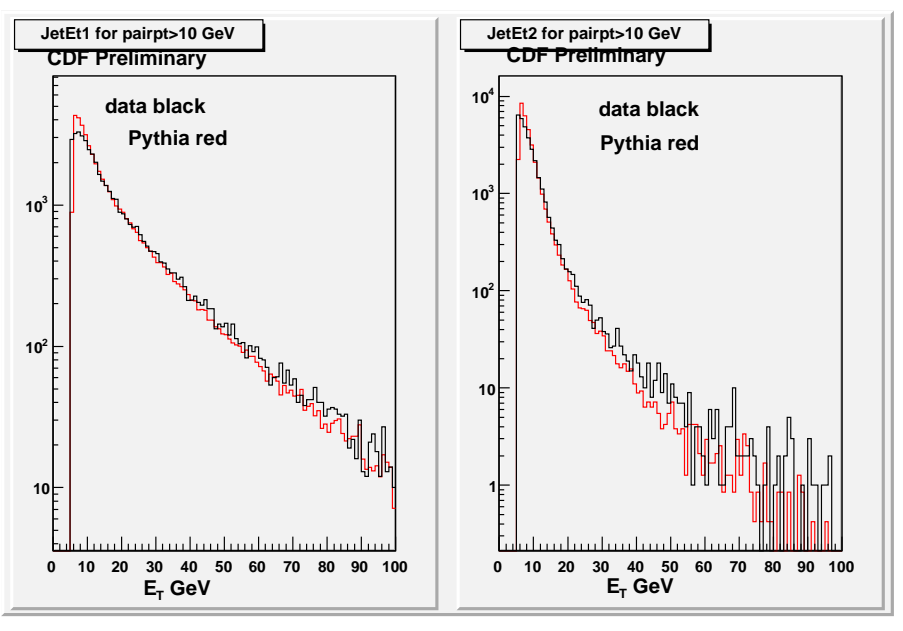

Figure 11: Left hand plot shows the recoil jet1 $\mathrm{E}_{\mathrm{T}}$ distribution for $\mathrm{p}_{\mathrm{TZ}}>10 \mathrm{GeV} / \mathrm{c}$, data and PYTHIA. Right hand plot is the same for jet2. The jets are ordered in decreasing $\mathrm{E}_{\mathrm{T}}$.

tut Naional de Physique Nucleaire et Physiques des Particules/CNRS; the Russian Foundation for Basic research; the Ministerio de Ciencia e Innovacion, Spain; the Slovak R\&D Agency; and the Academy of Finland. 


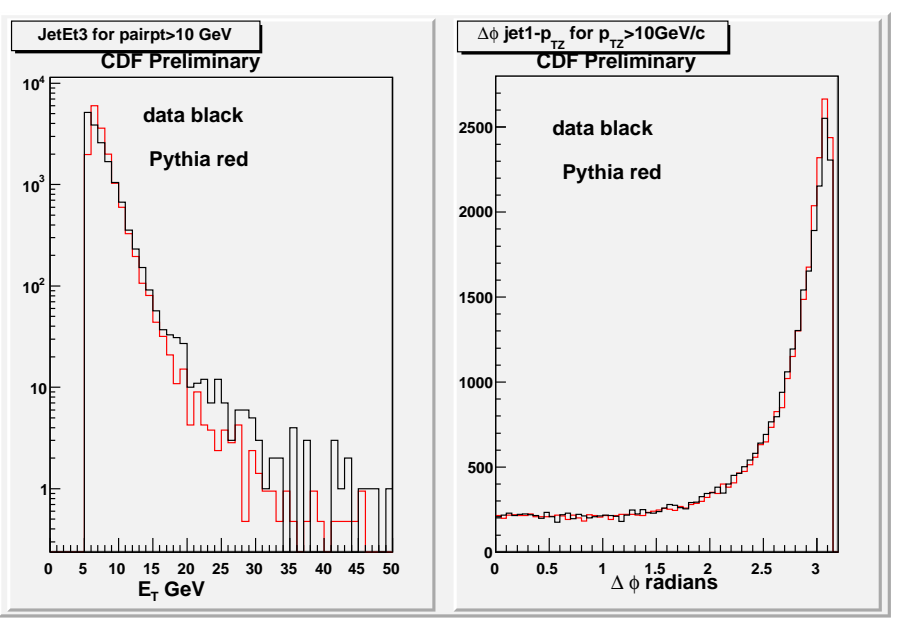

Figure 12: Left hand plot shows the recoil jet3 $\mathrm{E}_{\mathrm{T}}$ distribution for $\mathrm{p}_{\mathrm{TZ}}>10 \mathrm{GeV} / \mathrm{c}$, data and PYTHIA. Right hand plot is $\Delta \phi$ between jet1 and $\mathrm{p}_{\mathrm{TZ}}$. Note the strong recoil peak near $\Delta \phi=\pi$, and the long, rather flat tail.

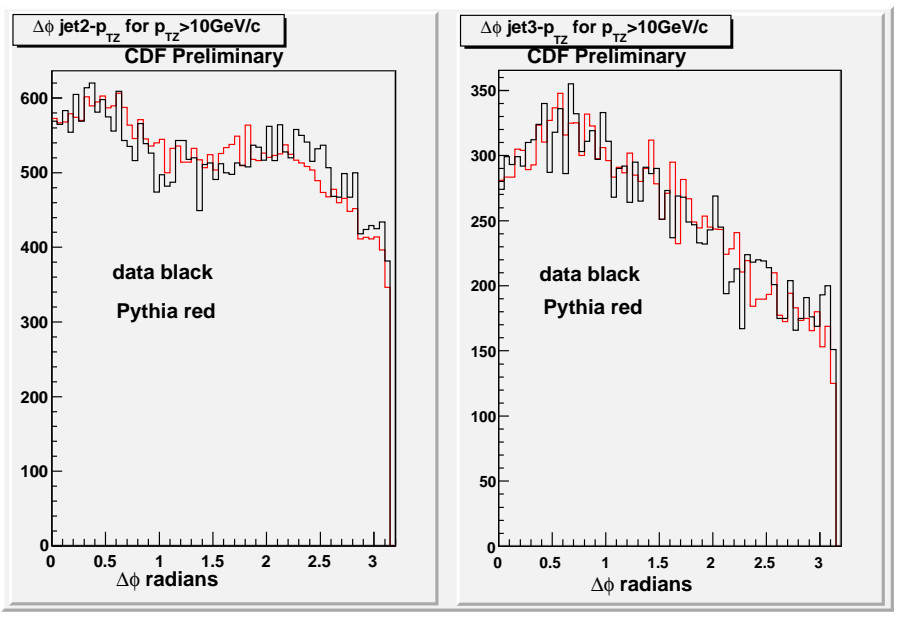

Figure 13: Left hand plot is $\Delta \phi$ between jet 2 and $\mathrm{p}_{\mathrm{TZ}}$, and the right hand plot is $\Delta \phi$ between jet 3 and $\mathrm{p}_{\mathrm{TZ}}$, all for $\mathrm{p}_{\mathrm{TZ}}>10 \mathrm{GeV} / \mathrm{c}$. PYTHIA agrees well with the data. The jets are not excluded from the transverse region. 


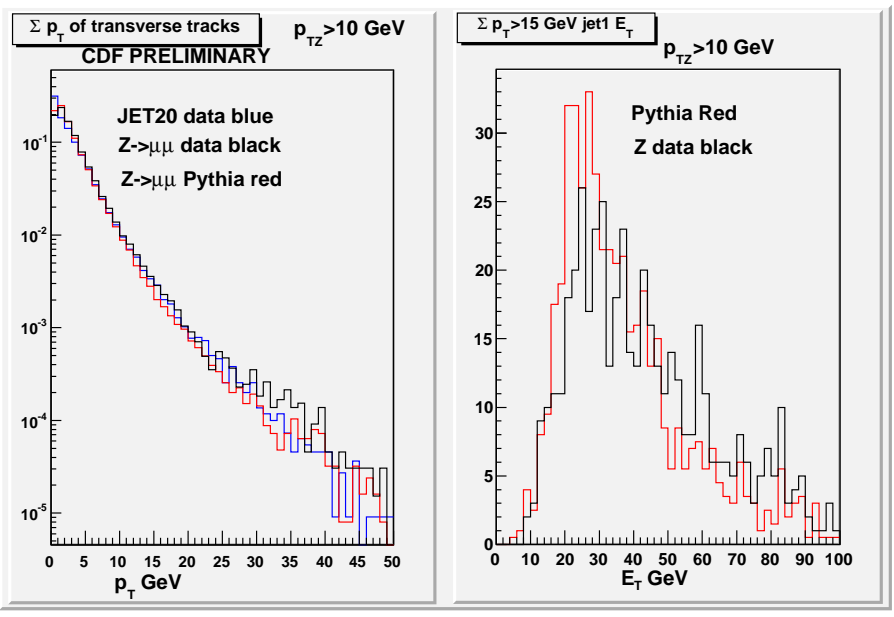

Figure 14: Left hand plot shows the $\Sigma$ transtrackp $\mathrm{p}_{\mathrm{T}}$ distribution for $\mathrm{p}_{\mathrm{TZ}}>10 \mathrm{GeV} / \mathrm{c}$ compared to jet20 data and PythiA Monte Carlo. The right hand plot is the leading jet $\mathrm{E}_{\mathrm{T}}$ distribution after the $\Sigma$ transtrackp $\mathrm{p}_{\mathrm{T}}>15$ $\mathrm{GeV} / \mathrm{c}$ requirement.

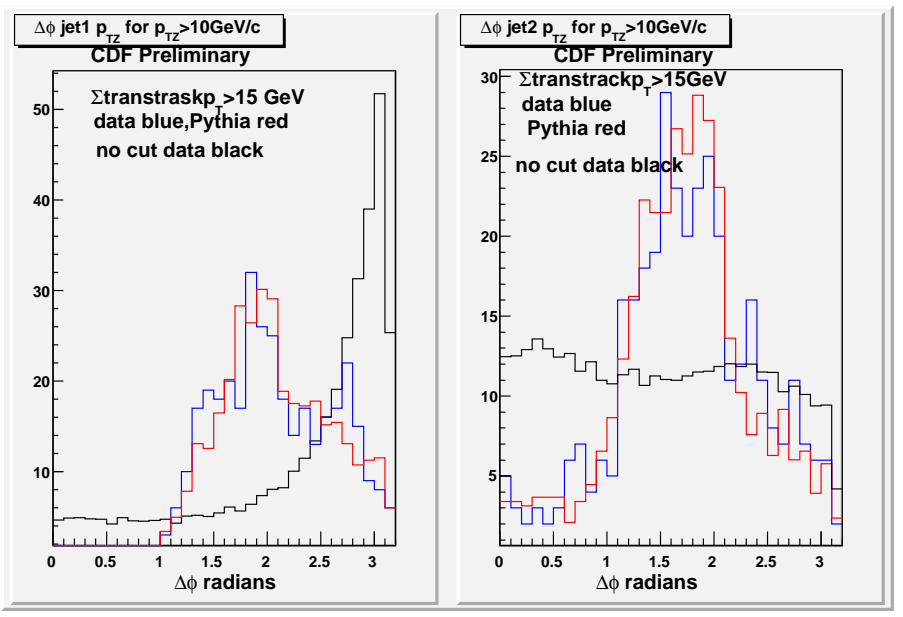

Figure 15: Left hand plot shows $\Delta \phi$ between jet1 and $\mathrm{p}_{\mathrm{TZ}}$, before and after the $\Sigma$ transtrackp $>15 \mathrm{GeV} / \mathrm{c}$ cut, compared to PYTHIA. The right hand plot is the same for $\Delta \phi$ between jet 2 and $\mathrm{p}_{\mathrm{TZ}}$. Note that both jets move into the transverse region. 


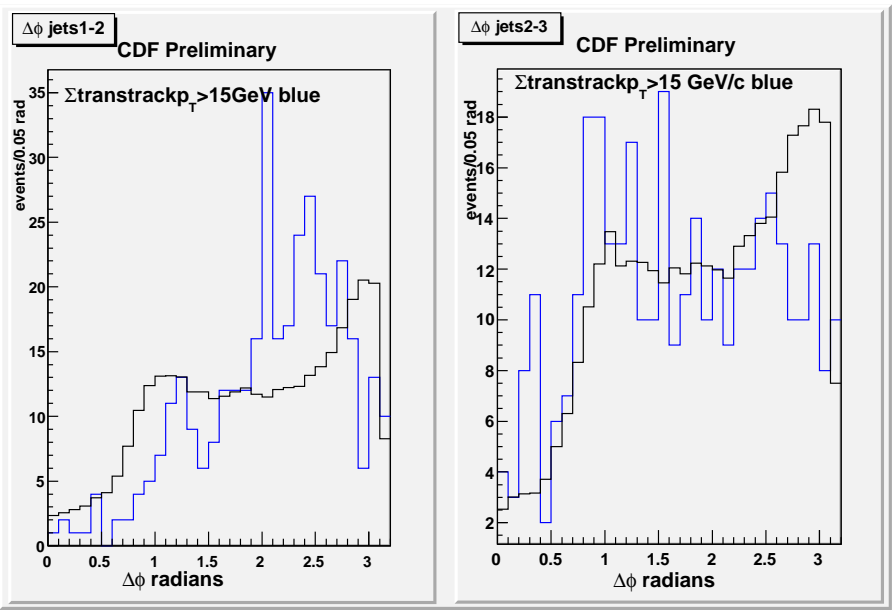

Figure 16: Left hand plot shows $\Delta \phi$ between jets 1 and 2 before and after the $\Sigma$ transtrackp $>15 \mathrm{GeV} / \mathrm{c}$ 'trigger' requirement. The before plot has been scaled by 0.011 to normalize to the same number of events. Note the peak in the after plot around 2.3 radians. The right hand plot is the same thing for jets 2 and 3 . DPI should show up as a peak near $\pi$, where none is observed.

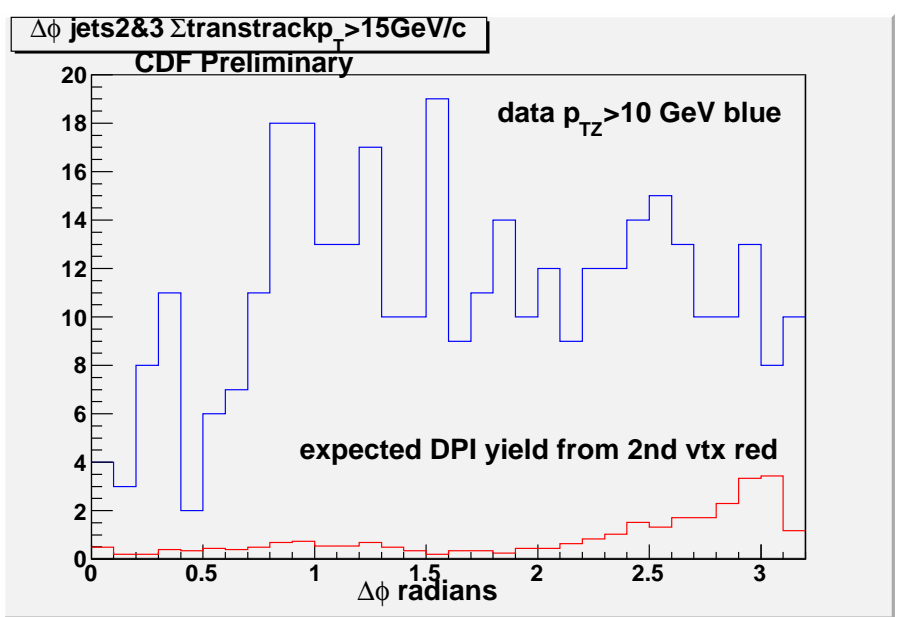

Figure 17: $\Delta \phi$ for jets 2-3 after the $\Sigma$ transtrackp $\mathrm{p}_{\mathrm{T}}>15 \mathrm{GeV} / \mathrm{c}$ compared to $\Delta \phi$ for jets $3-4$ on the second vertex, normalized as described in the text. For DPI in the Z sample, one of the 'jets' is the Z, so jet1 is the recoil, and jets 2 and 3 form the independent scatter. 


\section{References}

[1] T. Aaltonen et al.,(CDF Collaboration), Phys. Rev. D. 82,034001 (2010).

[2] T. Sjostrand et al., Comput. Phys. Commun. 135, 238 (2001). This analysis used Pythia 6.2.2.4.

[3] V.M.Abazov et al., (D0 Collaboration), Phys. Rev. D. 81052012 (2010).

[4] A. Abulencia et al.,(CDF Collaboration), J.Phys. G 342457 (2007).

[5] A. Bhatti et al.,Nucl. Instr. Meth. A 566375 (2006).

[6] M.M.Block and F. Halzen, Phys. Rev. D 72, 036006 (2005). These authors quote a fitted total cross section at $1.8 \mathrm{TeV}$ of $75 \mathrm{mb}$. Extrapolating to $1.96 \mathrm{TeV}$ and subtracting the elastic cross section of 16 $\mathrm{mb}$ gives $60 \mathrm{mb}$ inelastic.

[7] The bunch crossing frequency was constant during Tevatron Run 2. The peak luminosity was 4E32 $\mathrm{cm}^{-2} \mathrm{sec}^{-1}$, and $1 \mathrm{E} 32$ was fairly typical average over a 20 hour antiproton store. 